\title{
Vitrification of bovine blastocysts obtained by in vitro culture of oocytes matured and fertilized in vitro
}

\author{
M. Kuwayama, ${ }^{1}$ S. Hamano ${ }^{1}$ and T. Nagai ${ }^{2 *}$ \\ ${ }^{\prime}$ Tokyo Bio-Technology Center, Livestock Improvement Association 3-21-10 Higashi Shinagawa, \\ Shinagawa-ku, Tokyo 140, Japan; and ${ }^{2}$ National Institute of Animal Industry, Tsukuba \\ Norindanchi, Ibaraki 305, Japan
}

\begin{abstract}
Summary. Two experiments were conducted to assess the viability of bovine blastocysts obtained by in vitro fertilization of oocytes matured in vitro (IVM-IVF) and cryopreserved by vitrification. In Expt 1, the optimal concentrations of glycerol and 1,2propanediol in the basic medium (modified TCM199) for cooling and warming without formation of ice crystals were determined by plunging the solution into liquid nitrogen and then warming it in a water bath at $15^{\circ} \mathrm{C}$; when both glycerol and 1,2-propanediol were present in the solution $(>45 \% \mathrm{v} / \mathrm{v})$, vitrification of the medium was observed. In Expt 2, IVM-IVF blastocysts were equilibrated to the mixture of glycerol and 1,2propanediol $(0 \%$ to $45 \%)$ at $15^{\circ} \mathrm{C}$ in a stepwise manner as follows: (i) in one step, for $18 \mathrm{~min}$ to the final vitrification solution; (ii) in two steps, for $8 \mathrm{~min}$ in the first step and $10 \mathrm{~min}$ in the second step; (iii) in four steps, for $4 \mathrm{~min}$ in the first three steps and $6 \mathrm{~min}$ in the last step; (iv) in eight steps, for $2 \mathrm{~min}$ in each step, but $4 \mathrm{~min}$ in the last step; and (v) in 16 steps, for $1 \mathrm{~min}$ in each step, but $3 \mathrm{~min}$ in the last step. After removal of cryoprotectants, the blastocysts were cultured for $24 \mathrm{~h}$ in vitro. The survival rates for the embryos equilibrated in 1,2, 4, 8 and 16 step(s) were $56,89,100,100$ and $100 \%$, respectively. The blastocysts equilibrated in $1,2,4,8$ and 16 steps were vitrified by plunging the straws containing them into liquid $\mathrm{N}_{2}$, thawed and cultured in vitro. Higher survival rates were obtained for blastocysts equilibrated in 4, 8 and 16 steps $(79,82$ and $87 \%$, respectively) than for those in one $(0 \%)$ or two $(10 \%)$ steps. Ten blastocysts that survived after vitrification were transferred to ten recipients and six of these became pregnant. These results indicate that vitrification can be used for cryopreservation of blastocysts obtained by in vitro culture of IVM-IVF bovine follicular oocytes.
\end{abstract}

Keywords: in vitro; vitrification; fertilization; embryo transfer; cow

\section{Introduction}

The procedure for successful vitrification of mammalian embryos has been the subject of investigation, since Rall \& Fahy (1985) showed that mouse embryos can be successfully cryopreserved by vitrification. Scheffen et al. (1986) reported survival of mouse blastocysts after vitrification using a simple vitrification solution consisting of $25 \%$ glycerol and 25\% 1,2-propanediol.

However, in cattle, in vivo blastocysts did not survive after vitrification even though the solution used by Scheffen et al. (1986) and Massip et al. (1986) was used. Van der Zwalmen et al. (1989) reported that blastocysts did survive after vitrification, but only when they were equilibrated in three steps, in a glycerol sucrose solution instead of the solution used by Scheffen et al. (1986); the survival rate of blastocysts after vitrification was not high $(57 \cdot 1 \%)$.

*Reprint requests. 
Procedures of in vitro maturation (IVM) and in vitro fertilization (IVF) of bovine follicular oocytes have progressed to the extent that embryos can now be obtained by these techniques (Lu et al., 1987; Xu et al., 1987; Aoyagi et al., 1990; Eyestone \& First, 1989; Hamano \& Kuwayama, 1992). IVM-IVF embryos have been successfully frozen and thawed, resulting in pregnancies after embryo transfer (Goto et al., 1988; Kuwayama \& Hamano, 1991). There are no reports, however, on vitrification of the IVM-IVF blastocysts. In the present study, the viability of bovine blastocysts after vitrification was examined in vitro and in vivo.

\section{Materials and Methods}

\section{Experiment 1: vitrification assays}

The basic medium for the vitrification solution was TCM 199 (Earl's salts; Gibco, Grand Island, USA, modified with Hepes at $20 \mathrm{~mol}^{-1}$ and $0.35 \mathrm{~g}$ sodium bicarbonate $1^{-1}$ ) supplemented with $20 \%$ calf serum (CS; Gibco, Grand Island, USA). Because in a preliminary experiment, when IVM-IVF blastocysts were cultured for $24 \mathrm{~h}$ in phosphatebuffered saline (PBS; Nissui Pharmaceutical Co., Ltd, Tokyo, Japan) or in modified TCM199 (the basic medium) supplemented with $20 \% \mathrm{CS}$, the survival rate was higher $(P<0.05)$ with modified TCM199 $(96 / 104: 92 \%)$ than with PBS (79/100: 79\%). As a cryoprotectant, a mixture (equal volume) of glycerol and 1,2-propanediol was used. Transparent plastic straws $(0.25 \mathrm{ml}$; IMF, France) were filled with vitrification solution containing the mixture ( $10-60 \%$ ), sealed and plunged into liquid $\mathrm{N}_{2}$. The amorphous state (Boutron \& Kaufman, 1979) of the vitrification solution was checked by direct visual inspection as described by Scheffen $e t$ al. (1986). The straws were then warmed in a water bath at $15^{\circ} \mathrm{C}$. Devitrification occurring during warming was evident as whitening of the solution, giving the straw a milky aspect (Scheffen et al., 1986).

\section{Experiment 2: survival assays}

Source of blastocysts. Blastocysts were obtained by IVM-IVF of bovine follicular oocytes using the method described by Hamano \& Kuwayama (1992).

Collection of oocytes. Ovaries were obtained from a slaughterhouse and transported to the laboratory in $0.9 \%$ saline containing $0.1 \mathrm{mg}$ kanamycin $\mathrm{ml}{ }^{\prime}$ at $37^{\circ} \mathrm{C}$. All visible follicles $(2-5 \mathrm{~mm}$ in diameter) on the ovarian surface were aspirated with a $5 \mathrm{ml}$ syringe with an 18 -gauge needle.

Oocyte maturation in vitro. Cumulus-oocyte complexes were washed three times and cultured in a $\mathrm{CO}_{2}$ incubator $\left(2 \% \mathrm{CO}_{2}\right.$ in air with high humidity at $\left.38.5^{\circ} \mathrm{C}\right)$ for $20-21 \mathrm{~h}$ in droplets of maturation medium in culture dishes $(35 \mathrm{~mm} \times 15 \mathrm{~mm}$, Nunclon 153066; Inter-med, Denmark). The maturation medium used was TCM199 (Earl's salts with $25 \mathrm{mmol}$ Hepes 1 '; Gibco, USA) supplemented with $5 \%$ fetal bovine serum (Filtron Pty Ltd, Victoria) and antibiotics.

Sperm capacitation. Sperm capacitation was induced by the method described by Parrish et al. (1986). After thawing of frozen semen in a waterbath at $37^{\circ} \mathrm{C}$, the semen was suspended in $10 \mathrm{ml}$ of Brackett \& Oliphant (BO)

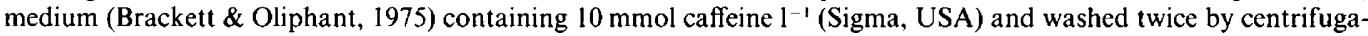
tion for $5 \mathrm{~min}$ at $800 \mathrm{~g}$. The concentration of live spermatozoa was adjusted to $2 \times 10^{7} \mathrm{cells}^{-1}$ and the sperm suspension was diluted twofold with BO medium containing $10 \mathrm{mg}$ bovine serum albumin $\mathrm{ml}^{-1}$ (Fraction V: Wako Pure Chem., Japan) and $10 \mu \mathrm{g}$ heparin $\mathrm{ml}^{-1}$ (Novo-heparin: Kodama Ind., Japan).

In vitro fertilization and culture. After maturation culture, the cumulus-oocyte complexes were washed by pipetting several times in the medium and introduced into the drops of sperm suspension. They were cultured together for $5 \mathrm{~h}$; the maturation medium from which the oocytes had been removed, although it still contained cumulus cells, was kept in a $\mathrm{CO}_{2}$ incubator.

All the inseminated oocytes, still surrounded by cumulus cells with attached motile spermatozoa, were subsequently introduced into the maturation medium that had been kept with the cumulus cells.

Forty-eight hours after insemination, the oocytes were removed from the cumulus cell masses by pipetting and then examined for their cleavage stages; four- to eight-cell embryos were further cultured with a cumulus cell monolayer in the same dish.

Embryos that had developed to the blastocyst (Fig. 1) and expanding blastocyst stage after 7 days of coculture with the cells were used for the experiments.

Survival assay after stepwise equilibration. IVM-IVF blastocysts were equilibrated to the mixture of glycerol and 1,2-propanediol $(0$ to $45 \%)$ at $15^{\circ} \mathrm{C}$ in a stepwise manner as follows: (i) in one step, for $18 \mathrm{~min}$ to the final vitrification solution; (ii) in two steps, for $8 \mathrm{~min}$ in the first step and $10 \mathrm{~min}$ in the second step; (iii) in four steps, for $4 \mathrm{~min}$ 


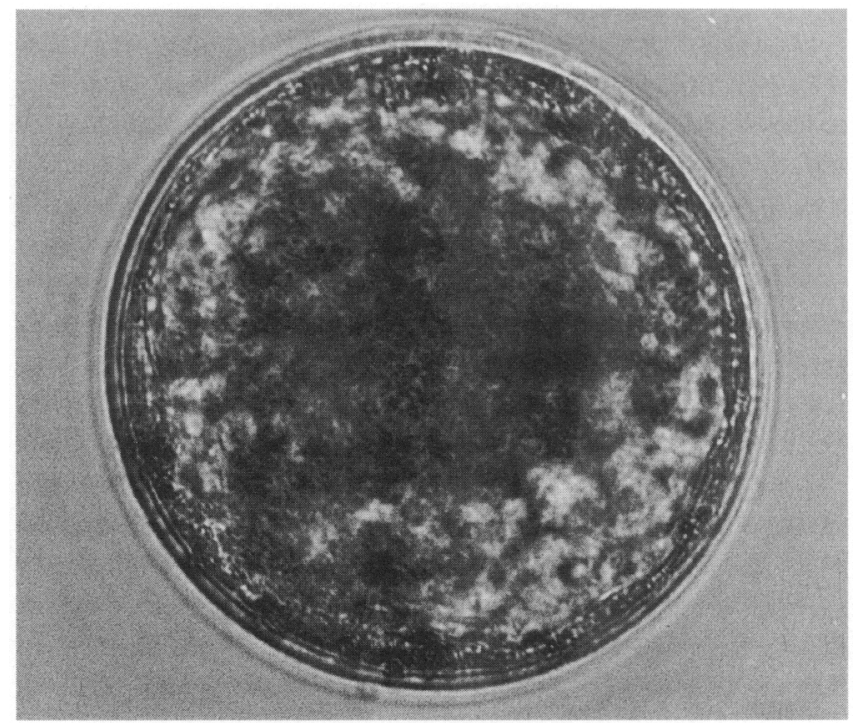

Fig. 1. Bovine blastocyst that was cultured with a monolayer of cumulus cells from the original oocytes for 7 days after in vitro fertilization of in vitro matured oocytes; $\times 300$.

in the first three steps and 6 min in the last step; (iv) in eight steps, for 2 min in each step, but 4 min in the last step; and (v) in $16 \mathrm{steps}$, in I min in each step, but for $3 \mathrm{~min}$ in the last step. Removal of cryoprotectants was carried out by exposing the embryos to a solution of $1 \mathrm{~mol}$ sucrose 1 ' in modified TCM199 in a one-step method (Scheffen et al., 1986). The embryos were then washed twice in the basic medium and cultured with a monolayer of cumulus cells for $24 \mathrm{~h}$ as described above. If the blastocysts expanded again after culture, the embryos were considered to have survived.

Survival assay after vitrification. The embryos that were equilibrated in the stepwise manner $(1,2,4,8$ and 16 steps $)$ as described above were loaded into a small column of the solution in $0.25 \mathrm{ml}$ transparent straws. All the straws were suspended above the liquid $\mathrm{N}_{2}\left(-40^{\circ} \mathrm{C}\right)$ for $30 \mathrm{~s}$ before plunging and storing for 3-7 days. Some embryos were also frozen by a one-step method (Leibo, 1984) that was known to be effective for cryopreservation of IVM-IVF embryos (Kuwayama \& Hamano, 199l). After thawing, they were used as control embryos for the embryo transfer.

Thawing was carried out by gentle shaking of the straw in a waterbath at $15^{\circ} \mathrm{C}$ for $8 \mathrm{~s}$. The contents of the straw were emptied into a solution of 1 mol sucrose $1^{-1}$ in modified TCM 199 and the embryos were placed on the surface of the medium and exposed therein for $10 \mathrm{~min}$ at $15^{\circ} \mathrm{C}$ to remove the cryoprotectants. The embryos were then washed twice in the basic medium and cultured with a monolayer of cumulus cells for $24 \mathrm{~h}$ as described above. If the blastocysts expanded again after culture, they were considered to have survived (Fig. 2).

The embryos that had survived after vitrification using 16-step equilibration were loaded in $0.25 \mathrm{ml}$ straws in the basic medium and transferred nonsurgically into ten synchronous recipients. Pregnancy was diagnosed by ultrasonic scanning when the cattle had passed 60 days without showing oestrus.

\section{Statistical analysis}

Data were analysed by $\chi^{2}$ tests.

\section{Results}

\section{Experiment 1}

When the vitrification solution contained glycerol and 1,2-propanediol, the concentration of cryoprotectant allowing the content of the straw to remain transparent during cooling as well as during warming was $>45 \%$. Thereafter, this concentration of cryoprotectants $(45 \%)$ was used in Expt 2. 


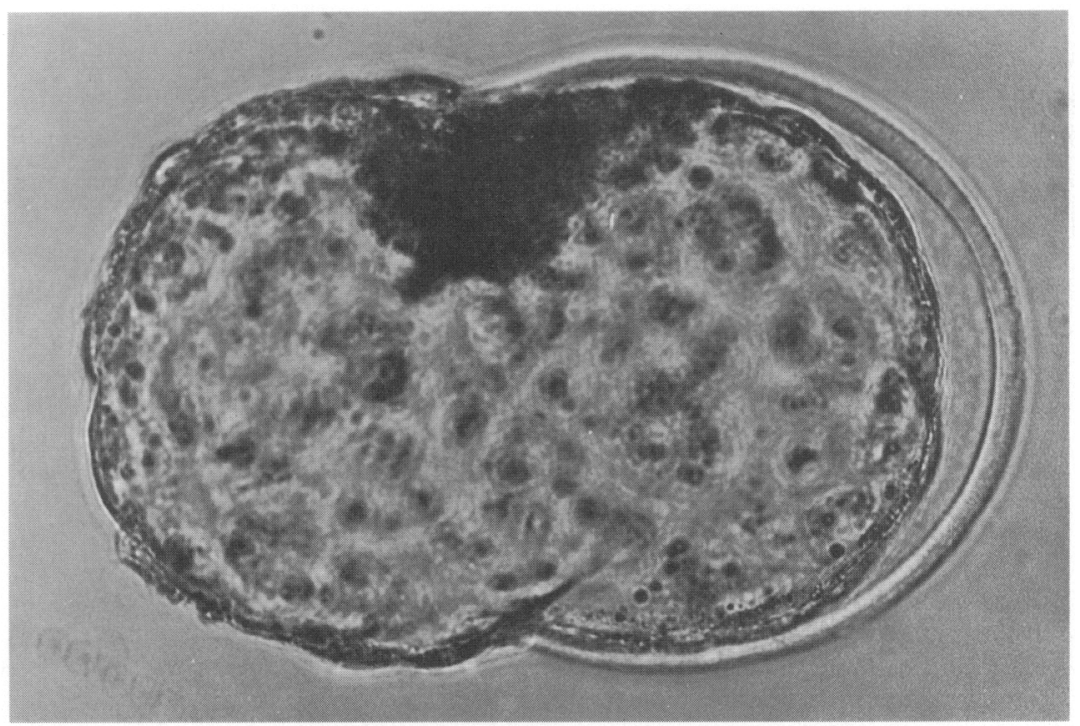

Fig. 2. Bovine blastocyst, from oocyte matured and fertilized in vitro, that was equilibrated in 16 steps to $22.5 \%$ glycerol and $22.5 \%$ 1,2-propanediol in modified TCM 199 , cryopreserved by vitrification and then cultured with a monolayer of cumulus cells for $24 \mathrm{~h} ; \times 300$.

\section{Experiment 2}

Higher survival rates were obtained for blastocysts equilibrated in four $(100 \%)$, eight $(100 \%)$ and 16 steps $(100 \%)$ than for those in one $(57 \%)$ or two steps $(89 \%)(P<0.05$, Table 1$)$.

Table 1. In vitro survival ${ }^{a}$ of bovine blastocysts after equilibration ${ }^{\mathrm{b}}$ in a stepwise manner

\begin{tabular}{lcc}
\hline $\begin{array}{l}\text { Equilibration } \\
\text { procedure } \\
\text { (number of steps) }\end{array}$ & $\begin{array}{c}\text { Number of } \\
\text { blastocysts } \\
\text { examined } \\
\text { (number of trials) }\end{array}$ & $\begin{array}{c}\text { Number (\%) of } \\
\text { blastocysts } \\
\text { survived } \\
\text { in vitro }\end{array}$ \\
\hline 1 & $50(3)$ & $28(56)^{\circ}$ \\
2 & $65(3)$ & $58(89)^{\mathrm{d}}$ \\
4 & $50(3)$ & $50(100)^{\circ}$ \\
8 & $50(3)$ & $50(100)^{\circ}$ \\
16 & $61(3)$ & $61(100)^{\circ}$ \\
\hline
\end{tabular}

"After equilibration and removal of cryoprotectants, blastocysts were cultured for $24 \mathrm{~h}$.

${ }^{b}$ Blastocysts were equilibrated in a stepwise manner to $22.5 \%$ glycerol $22.5 \%$ and 1,2-propanediol in modified TCM 199.

c.d."Values with different superscripts are significantly different $(P<0 \cdot 05)$.

When blastocysts were equilibrated in four, eight and 16 steps, the survival rate after vitrification was significantly higher $(79,82$ and $82 \%$, respectively) than when equilibrated in one $(0 \%)$ or two steps $(10 \%)(P<0 \cdot 05$, Table 2$)$.

When ten embryos that had survived after vitrification using 16-step equilibration and developed to the expanding blastocyst stage were transferred into ten synchronous recipients, six $(60 \%)$ were considered to be pregnant after diagnosis using ultrasonography 60 days after embryo 
clear, but osmotic injury during equilibration has been suggested to be lethal (Massip et al., 1989). In the present study, when blastocysts were equilibrated in one or two steps before vitrification, drastic distortion of zona pellucida and over-shrinkage of blastocysts occurred just after exposure to the solution, resulting in low percentages $(0-10 \%)$ of survival after vitrification, even if $56 \%$ (one step) and $89 \%$ (two steps) expanded again after removal of cryoprotectants without vitrification. By contrast, high proportions of blastocysts expanded again after vitrification when they were equilibrated in four, eight and 16 steps. In the 16-step method in particular, no distortion of zona pellucida occurred, shrinkage of blastocysts was slight and $87 \%$ of vitrified blastocysts expanded again. These results suggest that osmotic injury caused by water outflow might be avoided by increasing equilibration steps, resulting in high survival rates.

In a preliminary experiment, reducing the duration of exposure (to 3 or $10 \mathrm{~min}$ instead of $18 \mathrm{~min}$ ) of blastocysts to the final vitrification solution in one step did not improve the survival of blastocysts after vitrification. Furthermore, when blastocysts that had been equilibrated in 16 steps for $18 \mathrm{~min}$ were exposed to the final vitrification solution for an additional $5 \mathrm{~min}$, a high percentage of blastocysts expanded again after vitrification (data not shown). Thus, it is suggested that the vitrification solution used in the present study is not as toxic to blastocysts as suggested by Massip et al. (1986).

Pregnancies following transfer of in vivo embryos that were equilibrated in two steps and vitrified by the method used by Scheffen et al. (1986) have been reported in cattle (Massip et al., 1986; Douchi et al., 1990), but only late morulae and early blastocysts survived after vitrification, while blastocysts did not (Massip et al., 1986). Van der Zwalmen et al. (1989) reported that bovine in vivo blastocysts can survive after vitrification, but only when they are equilibrated in three steps, in a glycerol sucrose solution instead of the solution used by Massip et al. (1986), though the survival rate of the blastocysts after vitrification was not high $(57 \%)$ (Van der Zwalmen et al., 1989). When IVM-IVF blastocysts instead of in vivo blastocysts were vitrified by the methods of Massip et al. (1986) and Van der Zwalmen et al. (1989), the same results as they reported were obtained (data not shown). It therefore appears that the improvement in the survival of blastocysts reported by Van der Zwalmen et al. (1989) is due to an additional equilibration which reduces osmotic injury.

It has been shown that a mixture of $25 \%$ glycerol and $25 \%$ 1,2-propanediol in phosphatebuffered saline is the least toxic combination for mouse embryos, keeping the vitrification solution in an amorphous state (Scheffen et al., 1986). It can be concluded that $22 \cdot 5 \%$ glycerol $22 \cdot 5 \%$, 2 propanediol in modified TCM199 is effective not only for keeping the vitrification solution in an amorphous state but also for vitrification of bovine IVM-IVF blastocysts, when they are gradually equilibrated to the final vitrification solution.

We thank E. Fuku for useful advice and M. Takahashi, N. Takenouchi, K. Endo and M. Sato for excellent technical assistance.

\section{References}

Aoyagi, Y., Fukui, Y., Iwazumi, Y., Urakawa, M. \& Ono, H. (1990) Effects of culture systems on development of in vitro fertilized bovine ova into blastocysts. Theriogenology 34, 749-759.

Brackett, B.G. \& Oliphant, G. (1975) Capacitation of rabbit spermatozoa in vitro. Biology of Reproduction 12, $260-274$.

Boutron, P. \& Kaufman, A. (1979) Stability of the amorphous state in system water-1,2-propanediol. Cytobiology 16, 557-568.

Douchi, O., Takamura, H. \& Imai, K. (1990) Transfer of bovine embryos cryopreserved by vitrification. Japanese Journal of Animal Reproduction 36, 69-72.
Eyestone, W.H. \& First, N.L. (1989) Culture of early bovine embryos with oviduct tissue or in conditioned medium. Journal of Reproduction and Fertility 85, 415-420.

Goto, K., Kajihara, Y., Kosaka, S., Koba, M., Nakanishi, Y. \& Ogawa, K. (1988) Pregnancies after co-culture of cumulus cells with bovine embryos derived from in-vitro fertilization of in-vitro matured follicular oocytes. Journal of Reproduction and Fertility 83, $753-758$.

Hamano, S. \& Kuwayama, M. (1992) Effect of medium supplemented with bovine calf serum on the development of bovine oocytes matured and fertilized in 
vitro. Japanese Journal of Reproduction Technology 14, 6-16.

Kuwayama, M. \& Hamano, S. (1991) Cryopreservation of bovine blastocysts developed from in vitro fertilized oocytes matured in vitro. Japanese Journal of Animal Reproductive Technology 13, 165-171.

Leibo, S.P. (1984) A one-step method for direct nonsurgical transfer of frozen-thawed bovine embryos. Theriogenology 21, 767-789.

Lu, K.H., Gordon, I., Gallagher, M. \& McGoven, H. (1987) Pregnancy established in cattle by transfer of embryos derived from in vitro fertilization of oocytes matured in vitro. Veterinary Record 121, 259-260.

Massip, A., Van der Zwalmen, P., Scheffen, B. \& Ectors, F. (1986) Pregnancies following transfer of cattle embryos preserved by vitrification. Cryo-Letters 7, 270-273.

Massip, A., Van der Zwalmen, P., Scheffen, B. \& Ectors, F. (1989) Some significant steps in the cryopreservation of mammalian embryos with a note on a vitrification procedure. Animal Reproduction Science $19,117-129$.
Parrish, J.J., Susko-Parrish, J.L., Leibfried-Rutledge, M.L., Crister, E.S., Eyestone, W.H. \& First, N.L. (1986) Bovine in vitro fertilization with frozen thawed semen. Theriogenology 25, 591-600.

Rall, W.F. \& Fahy, G.M. (1985) Ice-free cryopreservation of mouse embryos at $-196^{\circ} \mathrm{C}$ by vitrification. Nature 313, 573-575.

Scheffen, B., Van der Zwalmen, P. \& Massip, A. (1986) A simple and efficient procedure for preservation of mouse embryos by vitrification. Cryo-Letters 7, $260-269$.

Van der Zwalmen, P., Touati, K., Ectors, F.J., Massip, A. \& Ectors, F. (1989) Vitrification of bovine blastocysts. Theriogenology 31, 270 (abstract).

Xu, K.P., Greve, T., Calleses, H. \& Hyttle, P. (1987) Pregnancy resulting from cattle oocytes matured and fertilized in vitro. Journal of Reproduction and Fertility 81, 501-504.

Received 30 July 1991 